\title{
Medicación potencialmente inapropiada en ancianos en un hospital de cuarto nivel en Bogotá
}

\author{
Esperanza Holguín ${ }^{1}$, José Gilberto Orozco ${ }^{2}$
}

\section{Resumen}

Introducción. La prescripción inapropiada de medicamentos es un problema reconocido, al cual los ancianos son una población vulnerable.

Objetivo. Describir la medicación potencialmente inapropiada en ancianos atendidos en consulta ambulatoria en un hospital de cuarto nivel de Bogotá en 2007.

Materiales y métodos. Se hizo un estudio de corte transversal. Como criterios de medicación potencialmente inapropiada en ancianos se utilizaron los propuestos por Beers, actualizados en 2002. La información se obtuvo de la última consulta registrada en la historia clínica en 2007, de una muestra de 420 ancianos que asistieron a consulta ambulatoria en un hospital de cuarto nivel.

Resultados. El promedio de medicamentos prescritos fue de 3,2. El 61,95\% de los pacientes recibía múltiples medicamentos. Los grupos de medicamentos más

1 Médica, especialista en Farmacología y Epidemiología; Grupo Red para el Uso Adecuado de los Medicamentos; profesora asistente de la Facultad de Medicina de la Pontificia Universidad Javeriana, Bogotá, D.C., Colombia.

2 Médico, especialista en Epidemiología, magíster en Farmacología, candidato a PhD en Salud Pública; Grupo Red para el Uso Adecuado de los Medicamentos; profesor asociado, Universidad Nacional de Colombia; profesor asistente de la Facultad de Medicina de la Pontificia Universidad Javeriana, Bogotá, D.C., Colombia. 
frecuentemente prescritos fueron aquellos con acción sobre el aparato cardiovascular (34,7\%), el aparato digestivo y el metabolismo (18,1\%), y el sistema nervioso (14,6\%). Según los criterios de Beers, $20,7 \%$ de los pacientes recibió medicación potencialmente inapropiada en ancianos. Se encontró asociación de dicha medicación con la administración de múltiples medicamentos. Hubo mayor frecuencia de medicación potencialmente inapropiada en ancianos con diagnósticos de enfermedades del aparato circulatorio y con la prescripción de fármacos que actúan sobre el sistema nervioso. A $15 \%$ de los pacientes se les prescribió medicamentos que pueden generar problemas no clasificados como medicación potencialmente inapropiada en ancianos por Beers.

Conclusiones. La medicación potencialmente inapropiada en ancianos es un problema frecuente. Es necesario adecuar y complementar los criterios de Beers a diferentes contextos.

Palabras clave: anciano, prescripción de medicamentos, salud pública, farmacoepidemiología, utilización de medicamentos.

\section{Title}

Potentially inappropriate medications in the elderly in a fourth level care hospital in Bogotá

\begin{abstract}
Background: The prescription of inappropriate medications is a recognized problem, to which the elderly are a vulnerable population.
\end{abstract}

Objective: To describe the potentially inappropriate medications in the elderly (PIME) treated at outpatient consultation in a fourth-level hospital in Bogota in 2007.

Materials and methods: A cross-sectional study was developed, updated in 2002 Beers criteria were used as PIME. The information was obtained from the last record in the medical history in 2007, from a sample of 420 outpatient elderly people in a fourth-level hospital.
Results: The average number of drugs prescribed was 3.2. $61.95 \%$ of the patients were polymedicated. Drug groups most frequently prescribed were those acting on the cardiovascular system $(34.7 \%)$, alimentary tract and metabolism $(18.1 \%)$ and nervous system (14.6\%). PIME was received by $20.7 \%$ of patients according to Beers criteria. PIME was associated with polypharmacy. PIME was more frequent in patients with diagnoses of circulatory system diseases and with action on the nervous system. Drugs that can cause problems not classified as PIME by Beers were prescribed to $15 \%$ of patients.

Conclusions: PIME is a frequent problem, it is necessary to adapt and supplement the Beers criteria to different context.

Key words: aged, prescription, public health, pharmacoepidemiology, drug utilization.

\section{Introducción}

El cambio en el perfil demográfico y de morbimortalidad, caracterizado por un incremento en la esperanza de vida y, por lo tanto, en el número de ancianos y de las enfermedades que predominan en ellos, se presenta como consecuencia de mejores condiciones de vida, un mejor conocimiento del funcionamiento del ser humano y, por consiguiente, una mayor posibilidad de intervención con medidas promocionales, preventivas, terapéuticas y de rehabilitación, entre las cuales los medicamentos juegan un papel importante[1].

Este cambio de perfiles se ha acompañado de una tendencia a considerar diversas condiciones de las personas como enfermedad y, por lo tanto, como merecedoras de tratamiento, general- 
mente con medicamentos[2]. La anterior tendencia va de la mano con la proliferación de nuevos medicamentos y ha contribuido a su uso frecuente e inapropiado. Según la Organización Mundial de la Salud (OMS), el 50\% de los medicamentos se prescribe, se dispensa y se consume de manera inadecuada[3].

La forma como se hacen actualmente la investigación y el desarrollo de nuevos medicamentos, en cuya orientación priman los intereses comerciales, resulta en un mercado anárquico que ofrece una inmensa cantidad de medicamentos nuevos con escasa novedad terapéutica real[4,5]. Esta proliferación desaforada de medicamentos conduce a que el conocimiento, la selección y el uso de los mismos sean innecesariamente más costosos y complejos, lo que contribuye a su uso inapropiado[6].

Otros factores contribuyen al problema de uso inapropiado de los medicamentos. Uno importante de resaltar es el predominio de la industria farmacéutica en la elaboración y difusión de la información sobre la utilidad y los riesgos asociados con el uso de los medicamentos, en las que utiliza diversas estrategias y técnicas publicitarias para promover sus ventas, con frecuencia pasando por encima de los intereses de la salud pública, lo que lleva a una sobrevaloración de la utilidad y subvaloración de los riesgos de los medicamentos, con el incremento consecuente de su consumo y la promoción de problemas como la medicalización de la vida (término utilizado por Illich[2] para referirse al proceso mediante el cual eventos y características de la vida cotidiana se convierten en asuntos médicos, hace parte de una crítica al creciente poder médico sobre los cuerpos y la vida de las personas), la automedicación y la polifarmacia[7,8].

Al extendido e inapropiado uso de medicamentos, se suma que en los procesos de investigación y desarrollo de nuevos medicamentos los estudios se realizan con pocos pacientes, durante periodos cortos y poco se incluyen los ancianos o personas con múltiples enfermedades y que reciben múltiples medicamentos; de esta manera, la eficacia, los efectos secundarios, los efectos a largo plazo o poco frecuentes y las interacciones entre medicamentos que se pueden presentar en los ancianos, son habitualmente poco conocidos cuando los medicamentos se usan en la práctica habitual[9].

Los ancianos son una población especialmente vulnerable ante el uso inapropiado de medicamentos, por tener una mayor frecuencia de enfermedad, existir una tendencia a considerar las condiciones de la vejez como enfermedad y sufrir de mayor abandono social, situaciones que se intenta resolver con asistencia médica, basada en la prescripción de medicamentos[10,11]. 
Igualmente, el anciano es más vulnerable a los efectos nocivos del uso inadecuado de los medicamentos por tener una menor reserva fisiológica y menor soporte social[10,11]. Esta mayor vulnerabilidad a los efectos nocivos y mayor propensión al uso inadecuado de medicamentos, señalan la necesidad de estudiar la magnitud, las causas y consecuencias de su uso inapropiado en los ancianos, para proponer intervenciones que contribuyan a enfrentar este problema.

Se han propuesto varias maneras de estudiar el uso inadecuado de medicamentos en los ancianos[12,13]. En 1991, Beers et al. desarrollaron los criterios explícitos de medicación potencialmente inapropiada en ancianos, que luego fueron actualizados en $1997 \mathrm{y}$ en 2002 mediante la técnica del consenso de expertos y de conceptos de medicina basada en "evidencias". Estos criterios son los más frecuentemente usados para estudiar la medicación potencialmente inapropiada en ancianos $[14,15]$.

La prevalencia de prescripción de este tipo de medicación es alta y ha sido bien documentada. Se ha evaluado en diferentes ámbitos, usando listas de criterios, frecuentemente basados en los de Beers, y se estima que afecta entre 12 y $60 \%$ de la población anciana[16-19].

Este reporte hace parte de un estudio mayor que incluyó pacientes que asistieron a consulta ambulatoria en un hospital de cuarto nivel y uno de primer nivel de atención. Se describen los patrones de prescripción de medicamentos y la medicación potencialmente inapropiada en ancianos atendidos en consulta ambulatoria, en un hospital de cuarto nivel en Bogotá durante 2007.

El objetivo del trabajo fue describir los patrones de prescripción en ancianos en dos instituciones de Bogotá durante el año 2007, evaluar la prevalencia de medicación potencialmente inadecuada en ancianos con base en los criterios de Beers, y explorar los posibles factores asociados, como edad, sexo y estrato socioeconómico del paciente, sexo del médico tratante, múltiple medicación, grupos de enfermedades diagnosticadas y grupos de medicamentos prescritos.

\section{Materiales y métodos}

Se llevó a cabo un estudio observacional descriptivo, de corte transversal.

\section{Población y muestra}

El total de pacientes mayores de 65 años atendidos durante el año 2007 en la consulta externa programada y de urgencias en un hospital de cuarto nivel de atención en Bogotá, fue de 16.593. Se calculó el tamaño de la muestra para determinar el porcentaje de medicación potencialmente inadecuada en ancianos; 
se asumió una prevalencia de $50 \%$, basada en la cifra propuesta por la OMS de medicación inadecuada[2], con un nivel de confianza de $95 \%$ y una precisión de $5 \%$, lo que resultó en un tamaño de muestra de 376 pacientes (Epi-Info 6).

Se tomó una muestra de 420 pacientes para los análisis exploratorios estratificados. Se hizo un muestreo aleatorio simple del total de pacientes de 65 ó más años registrados en la base de datos de atención, se numeraron los registros por orden de fecha de atención y se seleccionaron los correspondientes al listado de números aleatorios generado previamente, y se tomó la información de la última consulta médica registrada en la historia clínica que tenía la información relevante para el estudio durante el año 2007. Cuando no se encontró la información sobre las variables edad, diagnóstico y medicamentos en ninguna de las consultas del año 2007 del paciente seleccionado, se remplazó el registro para disminuir esta falla en las historias clínicas.

\section{Criterios y definiciones}

Se utilizaron como criterios de medicación potencialmente inadecuada en ancianos los desarrollados por Beers, que incluyen los fármacos que deberían evitarse en todos los ancianos y otros cuya prescripción depende de las condiciones particulares del anciano[14,15].

\section{Manejo de la información}

Las fuentes de información fueron los registros de historia clínica. Los datos se anotaron en un formulario diseñado para tal fin por una investigadora y por auxiliares de investigación previamente entrenados (estudiantes de medicina); la base de datos fue digitada en el programa Acces ${ }^{\circledR}$ y depurada antes de ser analizada por una investigadora. Los medicamentos se registraron de acuerdo con la clasificación anatomoterapéutica y química, y los diagnósticos, con base en la versión 10 de la Clasificación Internacional de Enfermedades (CIE 10). La clasificación de medicación potencialmente inadecuada en ancianos la hizo una investigadora, con base en los criterios de Beers actualizados en 2003[15].

\section{Resultados}

\section{Descripción general y demográfica}

De los 16.593 pacientes de 65 años o más atendidos en los servicios de consulta externa y urgencias de una Institución Prestadora de Servicios de Salud (IPS) de cuarto nivel en Bogotá durante el año 2007, se seleccionaron aleatoriamente 420 historias clínicas; de ellas, 249 $(59,3 \%)$ fueron de paciente de sexo femenino y $171(40,7 \%)$ masculino; 337 $(80,2 \%)$ fueron menores de 80 años y 83 $(19,8 \%)$ fueron mayores o de 80 años (gráfico 1). 


\section{Gráfico 1}

Distribución proporcional por edad y género

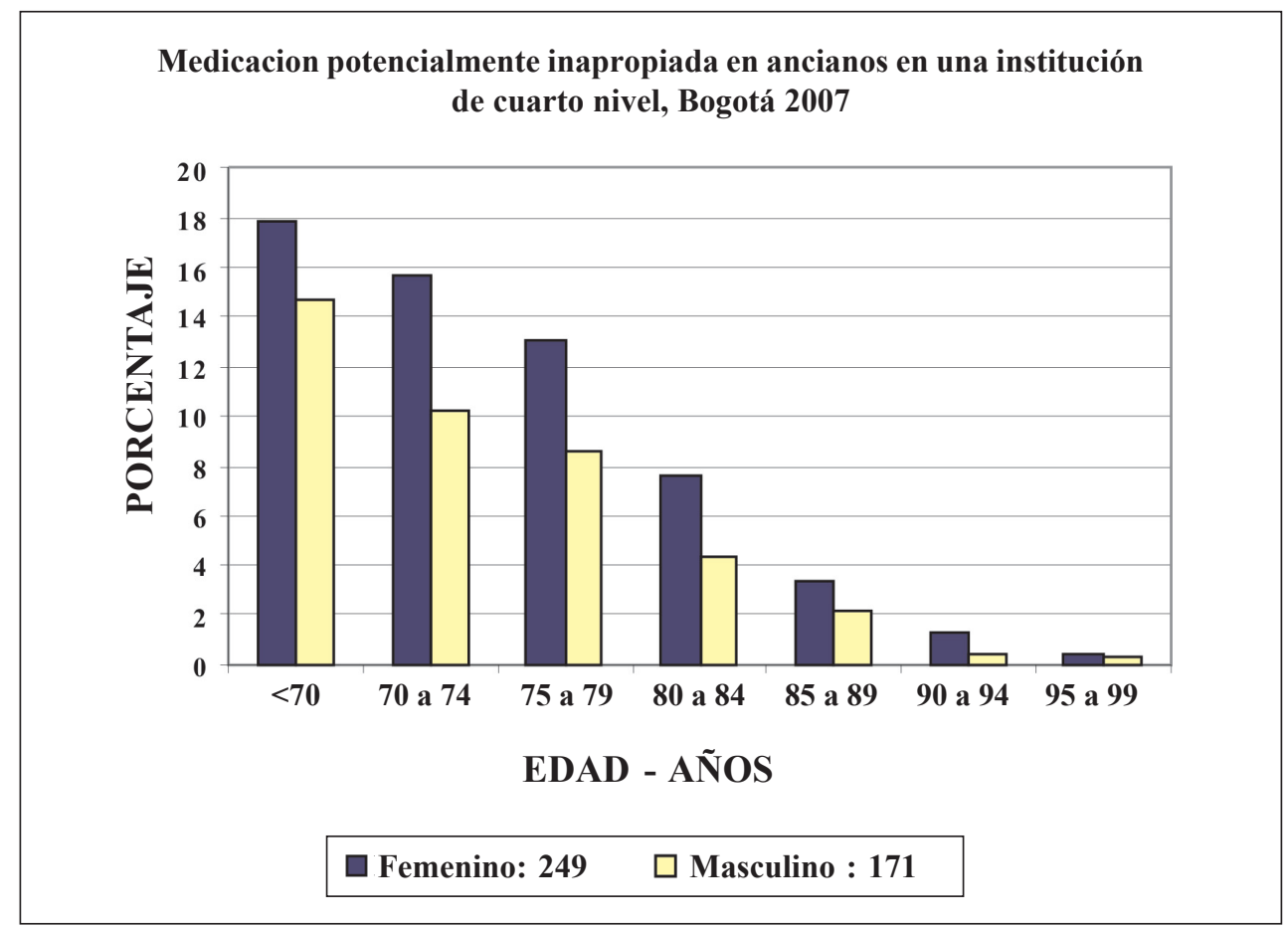

De los 420 pacientes, $230(54,8 \%)$ eran casados, 71 (16,9\%) viudos y 64 $(15,2 \%)$ solteros. En 408 (97,1\%) historias clínicas no se anotó el nivel de escolaridad. La ocupación se señaló en $188(44,8 \%)$ de las historias, la más frecuente fue las labores del hogar, 120 $(28,6 \%)$ de los 420 .

Se encontraron $89(21,2 \%)$ pacientes pensionados ( $\left.\mathrm{IC}_{95 \%} 17,38-21,32\right)$; de ellos, $40,4 \% \quad\left(\mathrm{IC}_{95 \%} 30,17-51,38\right)$ fueron mujeres y 59,6\% ( $\left.\mathrm{IC}_{95 \%} 48,62-69,83\right)$ fueron hombres.
Vivían en estrato socioeconómico 3 ó menor, $267(63,6 \%)$ pacientes $\left(\mathrm{IC}_{95 \%}\right.$ 57,77 - 68,18) (gráfico 2). Todos los pacientes atendidos pertenecían al régimen contributivo del Sistema General de Seguridad Social en Salud (SGSSS), y estaban asegurados por 16 Empresas Promotoras de Salud (EPS); se encontraron $297(70,7 \%)$ pacientes afiliados a cuatro EPS, incluido el seguro social $\left(\mathrm{IC}_{95 \%} 66,11-75,03\right)$. 


\section{Gráfico 2.}

Distribución de pacientes por estrato socioeconómico.

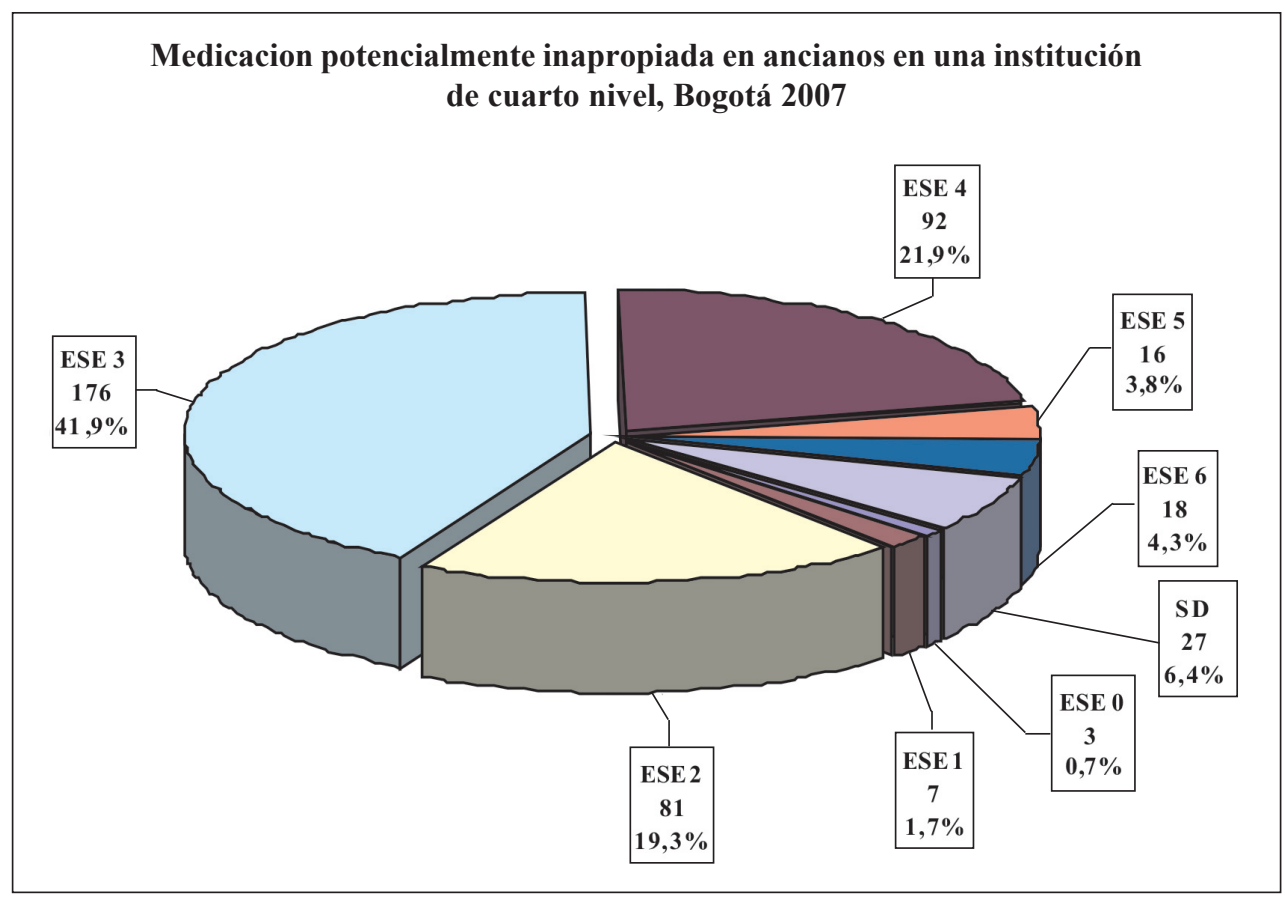

ESE: estrato socioeconómico.

\section{Descripción de la morbilidad}

Se hicieron 1.743 consultas médicas a los 420 pacientes durante el año 2007: moda, 1 ; mediana, 2; mínimo, 1 ; máximo, 58; promedio, 4,15 ( $\left.\mathrm{IC}_{95 \%} 3,64-4,66\right)$; desviación estándar, $5,381^{3}$; el 75\% de los pacientes asistió a cinco consultas médicas o menos en el año.
Se hicieron 1.329 diagnósticos en las consultas analizadas: mínimo, 1 ; máximo, 13; promedio, 3,16 $\left(\mathrm{IC}_{95 \%} 2,99-3,34\right)$. Los más frecuentes fueron enfermedades del aparato circulatorio, 404 diagnósticos $(30,4 \%)$, y enfermedades endocrinas, nutricionales y metabólicas, 242 diagnósticos (18,2\%) (gráfico 3).

3 A pesar de que las variables "número de consultas", "diagnósticos" y "medicamentos" son discretas y no tienen una distribución normal; se presentan los datos de promedio y desviación estándar con el fin de facilitar al lector su comparación con otros estudios que los presentan. 
Gráfico 3

Diagnósticos realizados - Clasificación CIE 10

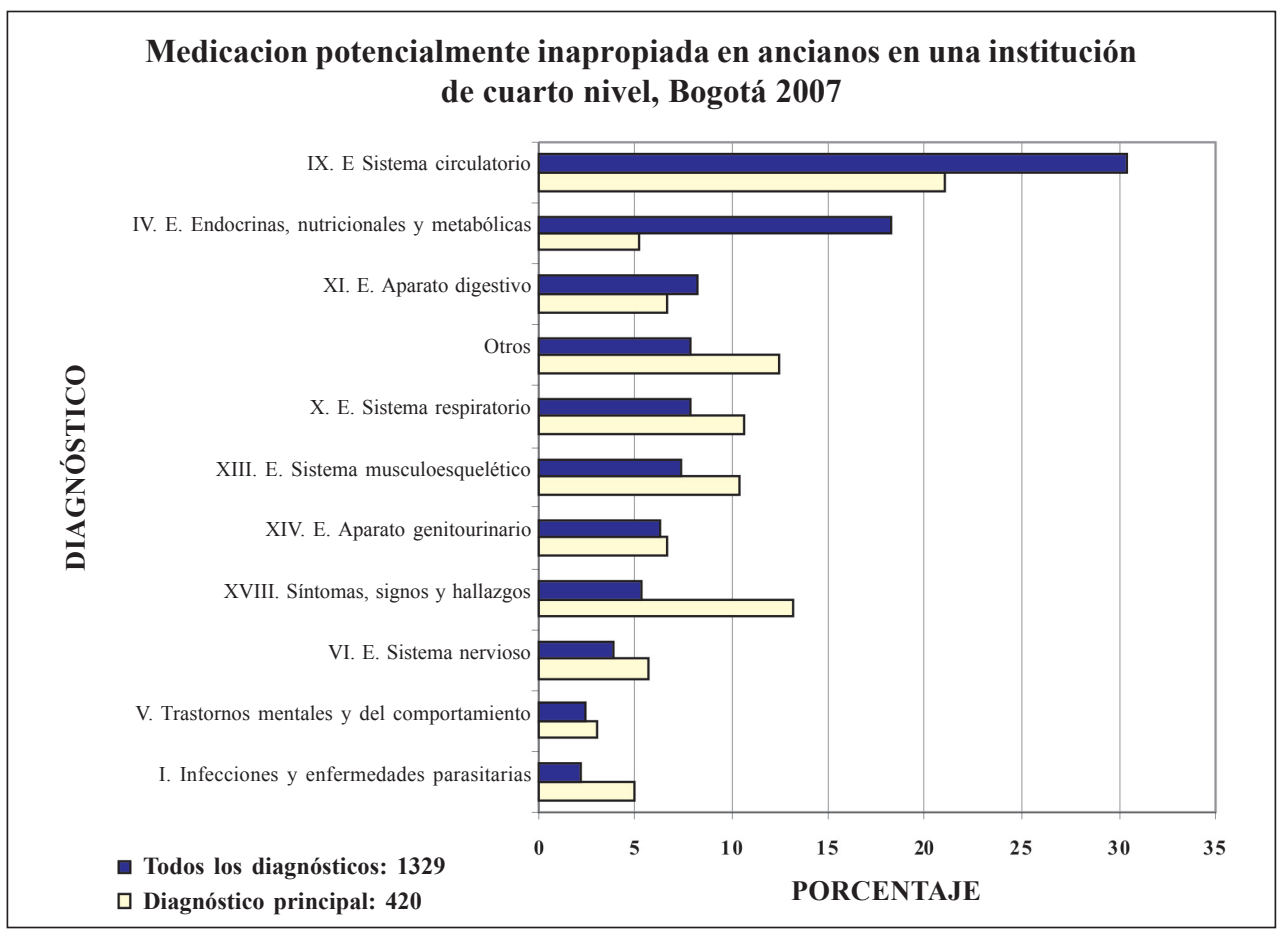

\section{Descripción del médico tratante}

De las consultas, $63,8 \%\left(\mathrm{IC}_{95 \%} 59,12\right.$ $68,30)$ fueron hechas por 268 médicos y $36,2 \%\left(\mathrm{IC}_{95 \%} 31,69-40,87\right)$, por 152 médicas. En la tabla 1 se presenta la distribución de las consultas analizadas, por servicio y especialidad.

\section{Descripción de la prescripción}

Se prescribieron 1.349 medicamentos en las consultas analizadas: mínimo, 0 ; máximo, 15 ; moda, 3 ; mediana, 4 ; promedio, 3,2 $\left(\mathrm{IC}_{95 \%}\right.$ 2,96-3,44); desviación estándar, 2,5386. Al incluir los antecedentes farmacológicos registrados con la última prescripción, se encontró que los pacientes estaban medicados con 2.067 medicamentos: mínimo, 0; máximo, 15; moda, 3; mediana, 5; promedio, 4,9 $\left(\mathrm{IC}_{95 \%} 4,62-5,18\right)$; desviación estándar, 2,9573 . En la consulta analizada se prescribieron cuatro o más medicamentos a 138 de los pacientes $\left(32,9 \%, \mathrm{IC}_{95 \%}\right.$ 28,49-37,46); al considerar tanto los medicamentos que se estaban consumiendo antes de la consulta como los prescritos en la consulta analizada, los que 
recibían varios medicamentos ascendieron a $256\left(61,95 \%, \mathrm{IC}_{95 \%} 56,22-65,55\right)$.

Los medicamentos más frecuentemente prescritos de acuerdo con la clasificación anatomoterapéutica y química, se agruparon así: 717 (34,7\%), del aparato cardiovascular; $375(18,1 \%)$, del aparato digestivo y metabolismo, y $301(14,6 \%)$, del sistema nervioso (tabla 2).

Tabla 1

Especialidad del médico tratante y servicio de atención

\begin{tabular}{|c|c|c|c|c|c|}
\hline Servicio & $\mathrm{n}$ & $\%$ & Especialidad & $\mathrm{n}$ & $\%$ \\
\hline \multirow[t]{11}{*}{ Consulta externa } & \multirow[t]{11}{*}{243} & \multirow[t]{11}{*}{57,9} & Familiar & 39 & 9,3 \\
\hline & & & Medicina interna & 39 & 9,3 \\
\hline & & & Cardiología & 24 & 5,7 \\
\hline & & & Gastroenterología & 21 & 5,0 \\
\hline & & & Nefrología & 16 & 3,8 \\
\hline & & & Neumología & 16 & 3,8 \\
\hline & & & Endocrinología & 12 & 2,9 \\
\hline & & & Neurología & 12 & 2,9 \\
\hline & & & Urología & 11 & 2,6 \\
\hline & & & $\begin{array}{l}\text { Clínica de dolor y cuidado } \\
\text { paliativo }\end{array}$ & 10 & 2,4 \\
\hline & & & Otros & 43 & 10,2 \\
\hline Consulta general urgencias & 122 & 29,0 & General & 122 & 29,0 \\
\hline \multirow{2}{*}{$\begin{array}{l}\text { Consulta urgencias e } \\
\text { interconsulta especialista }\end{array}$} & \multirow[b]{2}{*}{55} & \multirow[b]{2}{*}{13,1} & Medicina interna & 35 & 8,3 \\
\hline & & & Otros & 20 & 4,8 \\
\hline Total & 420 & 100 & & 420 & 100 \\
\hline
\end{tabular}


Tabla 2

Prescripción y medicación potencialmente inapropiada en ancianos

\begin{tabular}{|c|c|c|c|c|c|c|}
\hline \multicolumn{5}{|c|}{ Medicamentos - clasificación anatómica, terapéutica y química } & \multicolumn{2}{|c|}{ Inapropiados } \\
\hline Grupo anatómico & $\mathrm{n}$ & Código & $\begin{array}{l}\text { Subgrupo } \\
\text { terapéutico }\end{array}$ & $\mathrm{n}$ & $\mathrm{n}$ & $\%$ \\
\hline $\begin{array}{l}\text { Sistema digestivo y } \\
\text { metabolismo }\end{array}$ & 375 & $\begin{array}{l}\text { A01 } \\
\text { A03 }\end{array}$ & $\begin{array}{l}\text { Estomatológicos } \\
\text { Enfermedades } \\
\text { funcionales } \\
\text { gastrointestinales } \\
\text { Laxantes } \\
\text { Otros }\end{array}$ & $\begin{array}{r}11 \\
306\end{array}$ & $\begin{array}{r}1 \\
13\end{array}$ & 4,53 \\
\hline $\begin{array}{l}\text { Sangre y órganos formadores } \\
\text { de sangre }\end{array}$ & 161 & B01 & $\begin{array}{l}\text { Antitrombóticos } \\
\text { Otros }\end{array}$ & $\begin{array}{r}154 \\
7\end{array}$ & 5 & 3,11 \\
\hline Sistema cardiovascular & 717 & $\begin{array}{l}\mathrm{C} 01 \\
\mathrm{C} 08\end{array}$ & $\begin{array}{l}\text { Terapia cardiaca } \\
\text { Calcioantagonistas } \\
\text { Otros }\end{array}$ & $\begin{array}{r}35 \\
107\end{array}$ & $\begin{array}{l}9 \\
2\end{array}$ & 1,53 \\
\hline Sistema genitourinario & 13 & G04 & Urológicos & 9 & 1 & 7,69 \\
\hline $\begin{array}{l}\text { Antiinfecciosos de } \\
\text { uso sistémico }\end{array}$ & 96 & $\mathrm{~J} 01$ & $\begin{array}{l}\text { Antibacterianos } \\
\text { Otros }\end{array}$ & $\begin{array}{l}76 \\
20\end{array}$ & 5 & 6,58 \\
\hline Sistema musculoesquelético & 84 & $\begin{array}{l}\text { M01 } \\
\text { M03 }\end{array}$ & $\begin{array}{l}\text { Antiinflamatorios } \\
\text { Relajantes musculares } \\
\text { Otros }\end{array}$ & $\begin{array}{r}61 \\
5\end{array}$ & $\begin{array}{l}4 \\
5\end{array}$ & 10,71 \\
\hline Sistema nervioso & 301 & $\begin{array}{l}\text { N02 } \\
\text { N03 } \\
\text { N05 } \\
\text { N06 }\end{array}$ & $\begin{array}{l}\text { Analgésicos } \\
\text { Antiepilépticos } \\
\text { Sicolépticos } \\
\text { Sicoanalépticos } \\
\text { Otros }\end{array}$ & $\begin{array}{r}191 \\
20 \\
23 \\
57 \\
10\end{array}$ & $\begin{array}{r}5 \\
3 \\
2 \\
31\end{array}$ & 13,62 \\
\hline Sistema respiratorio & 181 & $\begin{array}{l}\mathrm{R} 03 \\
\mathrm{R} 06\end{array}$ & $\begin{array}{l}\text { Enf. obstructivas } \\
\text { de vía aérea } \\
\text { Antihistamínicos } \\
\text { Otros }\end{array}$ & $\begin{array}{r}150 \\
28 \\
3\end{array}$ & 3 & 6,08 \\
\hline Total & .067 & & & & 100 & 4,84 \\
\hline
\end{tabular}

De los 2.067 medicamentos prescritos, $100\left(4,8 \%\right.$; $\left.\mathrm{IC}_{95 \%}, 1,74-3,04\right)$ se clasificaron como medicación potencialmente inadecuada en ancianos según los criterios de Beers; estos fueron prescritos a 87 pacientes $(20,7 \%)\left(\mathrm{IC}_{95 \%}\right.$ $16,94-24,90)$. 
De los 100 medicamentos potencialmente inapropiados en ancianos, según los criterios de Beers, 94 (4,5\%) se consideraron de riesgo alto y $6(0,3 \%)$ de riesgo bajo, y 85 no tenían condiciones asociadas y 15 sí tenían condiciones asociadas (tabla 3$)$. En cinco (0,2\%) oca- siones en que los medicamentos prescritos pudieran haberse considerado potencialmente inadecuados en ancianos, según los criterios de Beers, no hubo información suficiente sobre la dosis o la duración del tratamiento para clasificarlos.

Tabla 3

Medicación potencialmente inapropiada según los criterios de Beers

Medicación potencialmente inapropiada en ancianos sin condiciones asociadas

\begin{tabular}{|c|c|c|c|c|}
\hline Grupo o fármaco & Problema & $\mathrm{n}$ & $\%$ & Gravedad \\
\hline Amitriptilina & $\begin{array}{l}\text { Por sus fuertes propiedades anticolinérgicas y } \\
\text { sedantes, rara vez es el antidepresivo de elección } \\
\text { en ancianos. }\end{array}$ & 18 & 18 & Alto \\
\hline $\begin{array}{l}\text { Fluoxetina } \\
\text { diariamente }\end{array}$ & $\begin{array}{l}\text { Fármaco de vida media larga y con riesgo de } \\
\text { producir estimulación excesiva del sistema } \\
\text { nervioso central, disturbios del sueño y agitación. } \\
\text { Existen alternativas seguras. }\end{array}$ & 12 & 12 & Alto \\
\hline $\begin{array}{l}\text { Antiespasmódicos } \\
\text { gastrointestinales: } \\
\text { hioscina }\end{array}$ & $\begin{array}{l}\text { Fármacos antiespasmódicos que tienen grandes } \\
\text { efectos anticolinérgicos y efectividad incierta. } \\
\text { Deben evitarse, especialmente a largo plazo. }\end{array}$ & 12 & 12 & Alto \\
\hline Difenhidramina & $\begin{array}{l}\text { Puede causar confusión y sedación. No debe } \\
\text { usarse como hipnótico y, cuando se use para } \\
\text { tratar emergencias alérgicas, debe hacerse en las } \\
\text { menores dosis posibles. }\end{array}$ & 8 & 8 & Alto \\
\hline Amiodarona & $\begin{array}{l}\text { Asociado con problemas del intervalo QT y } \\
\text { riesgo de torsades de pointes. No es eficaz en } \\
\text { ancianos. }\end{array}$ & 8 & 8 & Alto \\
\hline $\begin{array}{l}\text { Relajantes } \\
\text { musculares y } \\
\text { antiespasmódicos: } \\
\text { metocarbamol }\end{array}$ & $\begin{array}{l}\text { Muchos relajantes musculares y antiespasmó- } \\
\text { dicos son pobremente tolerados por pacientes } \\
\text { ancianos. Causan efectos adversos anticolinér- } \\
\text { gicos, sedación y debilidad. Además, su efectividad } \\
\text { a dosis toleradas por ancianos es cuestionable. }\end{array}$ & 6 & 6 & Alto \\
\hline Nitrofurantoína & $\begin{array}{l}\text { Puede producir daño renal. Hay alternativas } \\
\text { seguras disponibles. }\end{array}$ & 5 & 5 & Alto \\
\hline Clonidina & $\begin{array}{l}\text { Puede producir hipotensión ortostática y efectos } \\
\text { adversos en el sistema nervioso central. }\end{array}$ & 5 & 5 & Bajo \\
\hline
\end{tabular}


Tabla 3 (Continuación)

Medicación potencialmente inapropiada en ancianos sin condiciones asociadas

\begin{tabular}{llll}
\hline Grupo o fármaco $\quad$ Problema & $n \quad \% \quad$ Gravedad \\
\hline
\end{tabular}

Uso a largo plazo de dosis plenas de AINE no selectivos $\mathrm{COX}$ de vida media larga: naproxén

Uso a largo plazo de laxantes estimulantes: bisacodilo

Benzodiazepinas de acción larga: clordiazepóxido y diazepam

Nifedipina de corta acción

Dosis de benzodiazepinas de corta acción mayores a: $3 \mathrm{mg}$ de lorazepam

Anticolinérgicos y antihistamínicos: diversos antihistamínicos
Tienen potencial de producir sangrado gastrointestinal, falla renal, aumento de la presión arterial y falla cardiaca.

Puede exacerbar la disfunción intestinal.

$3 \quad 3 \quad$ Alto

Estos fármacos tienen una vida media larga en ancianos (a menudo varios días), produciendo sedación prolongada e incremento del riesgo de caídas y fracturas. Se prefieren las benzodiazepinas de acción corta e intermedia, si se requiere una benzodiazepina.

Pueden producir hipotensión y estreñimiento, por el incremento de la sensibilidad a las benzodiazepinas en ancianos.

Pequeñas dosis pueden ser efectivas y seguras. La dosis diaria total raramente excede las sugeridas.

Todos los antihistamínicos de no prescripción y algunos de prescripción pueden tener efectos anticolinérgicos potentes. Los antihistamínicos no anticolinérgicos se prefieren en ancianos para tratar reacciones alérgicas.

$3 \quad 3$ Alto

22 Alto

22 Alto

$\begin{array}{lll}0 & 0 & \text { Alto }\end{array}$

Total de medicación potencialmente inadecuada en ancianos sin $\quad 85 \quad 85$ condiciones asociadas
Medicación potencialmente inapropiada en ancianos con condiciones asociadas

Condición - Grupo o Problema

n $\%$ Gravedad fármaco 
Tabla 3 (Continuación)

Medicación potencialmente inapropiada en ancianos con condiciones asociadas

Condición - Grupo o Problema

n $\%$ Gravedad

fármaco

Daño cognitivo: es- Problema debido a alteraciones del SNC.

22 Alta

timulantes del SNC

EPOC:

Tienen efectos adversos en el SNC. Pueden

$2 \quad 2$ Alta

benzodiazepinas de

inducir depresión respiratoria. Pueden exacerbar

acción prolongada

o causar depresión respiratoria.

Obstrucción del flujo

Pueden producir poliuria y empeorar la

$1 \quad$ Alta

urinario: antidepresi-

incontinencia.

vos, descongestio-

nantes

Insomnio: teofilina.

Puede causar problema debido al efecto

$1 \quad 1 \quad$ Alta estimulante del SNC.

Enfermedad de Puede causar poblema debido a sus efectos $1 \quad 1 \quad$ Alta

Parkinson: metoclo- antidopaminérgicos/colinérgicos.

pramida

Depresión: benzodiazepinas de acción

Puede producir o exacerbar la depresión.

$1 \quad 1 \quad$ Alta

prolongada

Total MPIA sin condiciones asociadas

$15 \quad 15$

Total MPIA

100100

MPIA: medicación potencialmente inapropiada en ancianos; SNC: sistema nervioso central.

\section{Exploración de asociaciones}

No se encontró asociación entre medicación potencialmente inapropiada y edad, sexo o estrato socioeconómico del paciente, ni por el sexo del médico tratante.

Se encontró más prescripción potencialmente inadecuada en ancianos cuando los pacientes recibían cuatro o más fármacos (medicación múltiple), 65 de $256(25,39 \%)$, que cuando recibían menos de cuatro medicamentos, 22 de $164(13,41 \%)(\mathrm{p}=0,003131)$.

La mayor frecuencia de medicación potencialmente inadecuada se encontró en casos con diagnóstico de enfermedad cardiovascular: 15 de 404. Sin embargo, la mayor proporción se encontró en casos de diagnósticos de enfermedades del ojo y anexos: 7 de 29. Al considerar los pacientes con los diagnósticos más 
frecuentes, la proporción de medicación potencialmente inadecuada fue mayor en el grupo con enfermedades del aparato musculoesquelético: 11 de $99(11,1 \%), y$ fue menor en el grupo con diagnósticos del sistema endocrino, nutricional y metabólico: 2 en 242 pacientes $(0,83 \%)$ $(\mathrm{p}=0,000490)$ (tabla 4).

Tabla 4

Medicación potencialmente inapropiada y diagnósticos realizados

Clasificación de diagnósticos CIE 10*

\begin{tabular}{|c|c|}
\hline $\begin{array}{c}\text { Pacientes } \\
\text { con el } \\
\text { diagnóstico }\end{array}$ & $\begin{array}{l}\text { Paciente con } \\
\text { algún MPIA } \\
\text { por grupo de } \\
\text { diagnóstico }\end{array}$ \\
\hline$\%$ & $\%$ \\
\hline
\end{tabular}

I. Infecciones y enfermedades parasitarias

\begin{tabular}{rrrr}
29 & 6,9 & 7 & 24,1 \\
8 & 1,9 & 1 & 12,5 \\
242 & 57,6 & 2 & 0,8 \\
32 & 7,6 & 5 & 15,6 \\
52 & 12,4 & 9 & 17,3 \\
29 & 6,9 & 7 & 24,1 \\
404 & 96,2 & 15 & 3,7 \\
104 & 24,8 & 6 & 5,8 \\
109 & 26,0 & 6 & 5,5 \\
12 & 2,9 & 1 & 8,3 \\
99 & 23,6 & 11 & 11,1 \\
& & & \\
83 & 19,8 & 6 & 7,2 \\
70 & 16,7 & 7 & 10 \\
14 & 3,3 & 1 & 7,1 \\
16 & 3,8 & 3 & 18,8 \\
26 & 6,2 & & \\
\hline
\end{tabular}

III. Enfermedades de la sangre y del tejido hematopoyético

IV. Enfermedades endocrinas, nutricionales y metabólicas

V. Trastornos mentales y del comportamiento

VI. Enfermedades del sistema nervioso

VII. Enfermedades del ojo y los anexos

IX. Enfermedades del sistema circulatorio

X. Enfermedades sistema respiratorio

XI. Enfermedades del aparato digestivo

XII. Enfermedades de la piel y el tejido subcutáneo

XIII. Enfermedades del sistema musculoesquelético y del tejido conectivo

XIV. Enfermedades del aparato genitourinario

XVIII. Síntomas, signos y hallazgos clínicos y de laboratorio

XIX. Consecuencias de causas externas

XXI. Factores que influyen en la salud

Otros 
Al considerar los fármacos más frecuentemente prescritos, la proporción de medicación potencialmente inadecuada en ancianos fue mayor en el grupo con acción sobre el sistema nervioso: 41 de 301 (13,6\%), y fue menor en el grupo con acción sobre el aparato cardiovascular: en 11 de $717(1,5 \%)(\mathrm{p}=0,000000)$ (tabla 2).

Otro hallazgo importante de mencionar fue la prescripción de medicamentos que pueden generar problemas de inefectividad por interacción, de seguridad o por ser de dudosa o limitada eficacia, los cuales no están incluidos como medicación potencialmente inadecuada en ancianos en los criterios de Beers, se presentó en 78 ocasiones en 63 pacientes $\left(15 \%, \mathrm{IC}_{95 \%} 11,82-18,66\right)$ (tabla 5).

Tabla 5

Otra medicación potencialmente inapropiada en ancianos no incluida en los criterios de Beers

\begin{tabular}{lrr}
\hline Otros & $\mathrm{n}$ & $\%$ \\
\hline Más de un AINE en la misma & & \\
prescripción* & 7 & 1,7 \\
Alendronato* & 10 & 2,4 \\
Dipirona* & 9 & 2,1 \\
Glucosamina** $^{*}$ & 5 & 1,2 \\
Vitaminas** & 8 & 1,9 \\
Fármacos anti-Alzheimer** & 9 & 2,1 \\
Trimebutina** & 4 & 1,0 \\
AINE más antihipertensivo*** & 26 & 6,2 \\
\hline Total & 78 & 18,6 \\
\hline
\end{tabular}

* Potencial problema de seguridad.

** Dudosa o limitada eficacia.

*** Potencial problema de falta de efectividad.

\section{Discusión}

Las limitaciones más importantes de este estudio son las siguientes. En primer lugar, están las propias de los criterios de Beers, la mayoría señaladas por otros autores[16,17,20,21].

Entre ellas están: la dificultad para aplicarlos en diferentes ámbitos; que no cubran todas la posibilidades; que no incluyan la falta de prescripción de fármacos cuando están indicados; que se asuma que los diagnósticos son correctos; que no se tenga en cuenta que, en ocasiones, al presentarse falla o intolerancia de los fármacos de primera línea, es necesario usar otras alternativas que pueden considerarse potencialmente inadecuadas en ancianos; que no se consideren otros aspectos, como el cumplimiento de la prescripción o la automedicación, y que no permitan una aproximación a las causas de dicha medicación potencialmente inadecuada.

En segundo lugar, están las relacionadas con la calidad de la información registrada en las historias clínicas.

En tercer lugar, están las relacionadas con las características de la población fuente de la muestra, conformada por ancianos afiliados al régimen contributivo; para el 2003, en Colombia, 36,31\% de la población estaba afiliada a este régi$\operatorname{men}[22]$.

En cuarto lugar, están las relacionadas con el diseño del estudio: es un estudio 
descriptivo, sin pretensiones de establecer causalidad.

Las características sociodemográficas de la muestra representan la población que tiene acceso a la atención en el centro hospitalario seleccionado, caracterizada por pertenecer al régimen contributivo (en Bogotá, 73,2\%)[23] y proceder de estratos socioeconómicos en una distribución similar a la que existe en Bogotá[24].

El gran número de consultas al año y diagnósticos por paciente, y la mayor frecuencia de diagnósticos de enfermedades del aparato circulatorio y endocrinas, nutricionales y metabólicas, están de acuerdo con la situación de la población de 65 o más años de edad que presenta enfermedades múltiples y complejas, a lo que se suma la medicalización de la vida moderna, incluyendo la vejez. El tipo y la especialidad de la consulta médica practicada corresponden al nivel de atención de la institución estudiada.

La gran proporción de medicación múltiple y los medicamentos más frecuentemente prescritos encontrados en este estudio, se correlacionan con las características de la población anciana referidas en el párrafo anterior. Sin embargo, es necesario preguntarse si realmente es necesaria la medicación múltiple, dado que no existen pruebas que demuestren sus efectos benéficos; por el contrario, con frecuencia los estudios y los autores señalan sus riesgos e inconveniencia[8,19]. Además, es frecuente encontrar estudios que señalan que existe poco apego de los médicos a las guías de manejo[25]; por ejemplo, en este estudio se observa una elevada proporción de prescripción de medicamentos que actúan sobre el sistema renina-angiotensina-aldosterona, lo que no parece relacionado con las guías de manejo, lo que ha sido se ha demostrado en otros estudios en el caso de la hipertensión arterial[26,27].

El porcentaje de medicación potencialmente inadecuada en ancianos encontrado es importante $(20,7 \%)$ y está dentro de los rangos reportados en diversos estudios (14 a 60\%)[8,11-13,1620]; como es de esperarse, incluye los grupos de medicamentos y diagnósticos más frecuentes en este grupo de edad y se puede deducir que conduce a mayor sufrimiento humano (morbilidad adicional) y despilfarro de recursos por uso de medicamentos ineficaces y costos adicionales para manejar las reacciones adversas que se derivan[11,13,20,28,29].

En la exploración de asociaciones, no se encontraron diferencias en la medicación potencialmente inapropiada en los pacientes según edad, sexo, estrato socioeconómico, sexo y especialidad del médico tratante, lo que podría deberse a un problema de poder de la muestra, la que fue calculada para determinar la proporción de medicación potencialmente inadecuada en ancianos. 
Como lo han reportado otros autores, se encontró asociación entre la medicación potencialmente inadecuada en ancianos y la medicación múltiple[11,13,16,19,20,26,30]. También, se encontró mayor porcentaje de prescripción de medicamentos que actúan sobre los sistemas nervioso, musculoesquelético y respiratorio, y en los pacientes con diagnósticos de los aparatos musculoesquelético, respiratorio, digestivo y circulatorio, hallazgos que ayudan a orientar las áreas que se deben priorizar en futuros estudios e intervenciones, para mejorar el uso de los medicamentos en los ancianos.

Un resultado que merece especial reflexión es que la medicación potencialmente inadecuada en ancianos se presentó en porcentaje similar en el cuarto $(20,7 \%)$ y en el primer nivel de atención (21,51\%) (datos no presentados aquí). Mientras en el primero la mayoría de prescripciones fueron realizadas por especialistas, en el ámbito de un hospital universitario, en el segundo fueron realizadas por médicos generales, muchos de ellos recién egresados. Esto induce a pensar que en la medicación potencialmente inadecuada en ancianos poco influye la formación, información o educación de quienes los prescriben, e invita a profundizar en sus causas más allá de los conocimientos y capacidades de los individuos, y entenderla como un fenómeno social que es necesario estudiar en mayor profundidad.
Parece existir una "costumbre" de prescribir, en la cual el conocimiento científico de quien prescribe es un insumo que tiene poca influencia en la medicación potencialmente inadecuada en ancianos. Se hace necesario explorar otros factores que pueden determinar dicho tipo de medicación, como el papel de la industria farmacéutica en la educación médica, las condiciones habituales de la práctica médica, la influencia de la industria aseguradora en la prescripción, las condiciones de vida de los ancianos y la medicalización de la vejez, en fin, los factores sociales que determinan la prescripción de medicamentos en los ancianos.

En el 15\% de los pacientes la prescripción de medicamentos que pueden generar problemas de seguridad o ser de dudosa o limitada eficacia, y no clasificados como medicación potencialmente inadecuada en ancianos por Beers, muestra una limitación de los mismos y señala la necesidad de desarrollar iniciativas locales que, basadas en propuesta generales como la de Beers, propongan criterios de dicha medicación adaptados a cada realidad, útiles para evaluar y mejorar el uso de medicamentos en los ancianos.

Este estudio contribuye a demostrar que la medicación potencialmente inadecuada en ancianos es un problema en el medio colombiano, que es necesario caracterizar mejor, tanto desde el punto 
de vista de su magnitud, como de sus causas y consecuencias, para proponer, implementar y evaluar intervenciones que contribuyan a un mejor aprovechamiento de los medicamentos en los ancianos.

\section{Agradecimientos}

Los autores agradecen al Hospital Universitario San Ignacio, por las facilidades prestadas para la recolección de la información para este estudio.

\section{Conflictos de interés}

Los autores manifiestan no tener conflictos de intereses distintos del reconocimiento académico que puedan obtener por la publicación del manuscrito.

\section{Financiación}

Este estudio recibió apoyo financiero de la División de Investigaciones de la Universidad Nacional de Colombia y fue desarrollado parcialmente como parte de la jornada de trabajo de los investigadores en sus respectivas universidades.

\section{Bibliografía}

1. Comisión de Derechos de Propiedad Intelectual, Innovación y Salud Pública de la OMS. Salud pública, innovación y derechos de propiedad intelectual: informe de la Comisión de Derechos de Propiedad Intelectual, Innovación y Salud Pública. Suiza: OMS. 2006.
2. Illich I. Némesis médica. México D.F.: Editorial Joaquín Mortiz S. A. 1978.

3. OMS. Promoción del uso racional de medicamentos: componentes centrales. Perspectivas políticas sobre medicamentos de la OMS. 2002;5:1-6.

4. Angell M. La verdad acerca de la industria farmacéutica. Cómo nos engaña y qué hacer al respecto. Bogotá: Editorial Norma S.A. 2006.

5. Hubbard B. Secretos de la industria farmacéutica. Efectos adversos de muchos medicamentos: bajo sospecha su fabricación y prescripción. Barcelona: Terapias verdes. 2007.

6. World Health Organization. The selection and use of essential medicines. Report of the WHO Expert Committee. WHO technical report series 920 . Geneva: WHO. 2003.

7. Norris P, Herxheimer A, Lexchin J, Mansfield P. Drug promotion: What we know, what we have yet to learn. Ginebra: World Health Organization and Health Action International. 2005.

8. Blasco F, Martínez J, Villares P, Jiménez A. El paciente anciano polimedicado: efectos sobre su salud y sobre el sistema sanitario. Inf Ter Sist Nac Salud. 2005;29:152-62.

9. Laporte JR, Tognoni G. Principios de epidemiología del medicamento. Segunda edición. Barcelona: Ediciones científicas y técnicas Masson-Salvat; 1993.

10. Shah R. Drug development and use in the elderly: Search for the right dose and dosing regimen. $\mathrm{Br} J$ Clin Pharmacol. 2004;58:452-69. 
11. Milton JC, Hill-Smith I, Jackson SHD. Prescribing for older people. BMJ. 2008;336:606-9.

12. McLeod PJ, Huang AR, Tamblyn RM, Gayton DC. Defining inappropriate practices in prescribing for elderly people: A national consensus panel. Canadian Medical association Journal CMAJ. 1997;156:385-91.

13. Roth MT, Weinberger M, Campbell WH. Measuring the quality of medication use in older adults. Journal of the American Geriatrics Society. 2009;57:1096-102.

14. Beers MH. Explicit criteria for determining potentially inappropriate medication use by the elderly: An update. Arch Intern Med. 1997;157: 1531-6.

15. Fick DM, Cooper JW, Wade WE, Waller JL, Maclean JR, Beers MH. Updating the Beers criteria for potentially inappropriate medication use in older adults: Results of a US consensus panel of experts. Arch Intern Med. 2003;163:2716-24.

16. Niwata S, Yamada Y, Ikegami N. Prevalence of inappropriate medication using Beers criteria in Japanese longterm care facilities. BMC Geriatrics. 2006;6:1.

17. Ryan C, O'Mahony D, Kennedy J, Weedle P, Barry P, Gallagher P, et al. Appropriate prescribing in the elderly: An investigation of two screening tools, Beers criteria considering diagnosis and independent of diagnosis and improved prescribing in the elderly tool to identify inappropriate use of medicines in the elderly in primary care in Ireland. $J$ Clin Pharm Ther. 2009;34:369-76.
18. Corsonello A, Pedone C, Lattanzio F, Lucchetti M, Garasto S, Di Muzio M, et al. Potentially inappropriate medications and functional decline in elderly hospitalized patients. Journal of the American Geriatrics Society 2009;57:1007-14.

19. Gavilán E, Morales M, Hoyos JA, Pérez AM. Polimedicación y prescripción de fármacos inadecuados en pacientes ancianos inmovilizados que viven en la comunidad. Atención Primaria. 2006;38:476-82.

20. Fialová D, Onder G. Medication errors in elderly people: Contributing factors and future perspectives. $\mathrm{Br} \mathrm{J}$ Clin Pharmacol. 2009;67:641-5.

21. Soares MA, Fernández-Llimós F, Lança C, Cabrita J, Morais JA. Operacionalização para Portugal: Critérios de Beers de medicamentos inapropriados nos doentes idosos. Acta Med Port. 2008;21:441-52.

22. DANE. Encuesta de calidad de vida 2003. Bogotá: Departamento Nacional de Planeación. 2003.

23. DANE. Encuesta de calidad de vida para Bogotá 2007. Bogotá: Alcaldía Mayor de Bogotá, D.C. 2008.

24. DANE. Encuesta Nacional de Hogares 2004. Bogotá: Departamento Nacional de Planeación. 2004.

25. Strom B. Pharmacoepidemiology. Fourth edition. England: John Wiley \& Son Ltda. 2005.

26. Vara L, Sangrador A, Muñoz P, Sanz S. Uso de antihipertensivos en Cantabria (1995-2002): el desfase con las evidencias. Gac Sanit. 2004;18:406-8. 
27. Abaci A, Kozan O, Oguz A, Sahin M, Deger N, Senocak H, et al. Prescribing pattern of antihypertensive drugs in primary care units in Turkey: Results from the TURKSAHA study. Eur J Clin Pharmacol. 2007;63:397-402.

28. Passarelli MC, Jacob-Filho W, Figueras A. Adverse drug reactions in an elderly hospitalized population: Inappropriate prescription is a leading cause. Drugs Aging. 2005;22:767-77.
29. Chrischilles EA, van Gilder R, Wright K, Kelly M, Wallace RB. Inappropriate medication use as a risk factor for selfreported adverse drug effects in older adults. Journal of the American Geriatrics Society. 2009;57:1000-6.

30. Laroche ML, Charmes JP, Nouaille Y, Picard N, Merle L. Is inappropriate medication use a major cause of adverse drug reactions in the elderly? Br J Clin Pharmacol. 2006;63:177-86. 